\title{
Instrumentalisierung ärztlicher Experten
}

\section{Rainer M. Kaelin}

Dr. med., Facharzt für Pneumologie und Innere Medizin, Morges, vormals Vizepräsident der Lungenliga Schweiz und der Lungenliga Waadt

Der Begriff «Treu und Glauben» umschreibt die Tatsache, dass das Zusammenleben der Menschen auf gegenseitigem Vertrauen beruht. Tausch, Handel, Vermittlung von Information oder Gefühlsäusserungen setzen voraus, dass alle Beteiligten an einer ehrlichen Transaktion interessiert sind, die nicht durch Täuschung, verborgene Nebenabsichten oder Verschleierung von Fakten eine der Parteien übervorteilt.

Dieses Paradigma menschlicher Beziehungen wird komplexer, wenn die Transaktion zwischen zwei Parteien eine weitere einbezieht, die man mit dem Begriff «öffentliches Interesse» bezeichnen könnte. Ärzte, die zunächst das Wohl ihrer individuellen Patienten im Auge haben, bekommen eine vielschichtigere Rolle, sobald sie als Spitalärzte, akademische Lehrer, Experten oder als Beauftragte des öffentlichen Gesundheitswesens praktizieren. Dort können sie Kosten verursachen, welche die Gemeinschaft der Versicherten, der Staat oder die Gesellschaft zu tragen haben.

Der erste Teil der folgenden Überlegungen handelt von der ärztlichen Expertise im Medikamentenmarkt und in der öffentlichen Gesundheit. Der zweite Teil handelt vom Schweigen der Experten in der öffentlichen Debatte über strukturelle Massnahmen zur Kontrolle des Tabakkonsums und von den Schlussfolgerungen, die sich daraus ergeben.

\section{Der Fall Matsubara}

Eine ausführlichere französische Version des Artikels wird im Juni in Bioethica Forum erscheinen.

Eine Stellungnahme von Interpharma, dem Verband der forschenden pharmazeutischen Firmen der Schweiz, findet sich im Anchluss an den Beitrag.

Die Literaturangaben finden sich unter www.saez.ch $\rightarrow$ Aktuelle Ausgabe oder $\rightarrow$ Archiv $\rightarrow 2015 \rightarrow 18$.
Im Frühling 2013 musste der Kardiologe Hiroaki Matsubara von seiner akademischen Stelle an der Universität Kyoto zurücktreten, weil er Studienresultate gefälscht hatte. Sie sollten die Überlegenheit des Medikamentes Diovan ${ }^{\circledR}$ von Novartis gegenüber Konkurrenzprodukten bei zerebrovaskulären Insulten beweisen. Die Untersuchung förderte 34 Fälschungen zutage. Die Medien konzentrierten sich wie üblich auf die Verfehlungen des Forschers, der aus Eigennutz gehandelt hatte. Aus Sicht des «öffentlichen Interesses» sollte man jedoch auch weitere Aspekte betrachten:

- Die Täuschung bedeutet eine Rufschädigung der akademischen Institution und des Spitals, aber auch der Unbescholtenheit der Mitarbeiter, die alle in "Treu und Glauben» gehandelt hatten.

- Vertrauen, das die Gesellschaft und staatliche Behörden in Institutionen setzt, die dem Gemeinwohl dienen - wie Spitäler oder Universitäten aber auch in Experten, wird beeinträchtigt. «Treu und Glauben» werden verletzt.

- Würde die Täuschung nicht aufgedeckt, wäre zwar kein Vertrauensverlust, aber konkreter Schaden die Folge: Pharmavertreter würden Ärzte weltweit mit "wissenschaftlichen» Argumenten überzeugen, ihren Patienten das Medikament $\mathrm{X}$ zu verschreiben. Und Aufsichtsbehörden würden für fiktive Vorteile dem Produkt X höhere Preise zugestehen müssen, die von den Gesundheitssystemen, letztlich von der Allgemeinheit, zu tragen wären.

\section{Betrug als Strategie der Pharmaunter- nehmen? Die Mauer des Schweigens}

Professor Peter C. Goetsche vom Cochrane Centre in Kopenhagen wollte abschätzen, in welchem Ausmass Pharmafirmen betrügerisch handeln [1]*. Er suchte im Internet nach Fällen, indem er in die Suchmaschine die Kombination der Namen der zehn grössten Pharmafirmen und des Wortes Betrug (fraud) eingab. Von den zahlreichen Resultaten hat er jeweils den wichtigsten Fall der ersten Seite jeder der zehn Suchrunden genauer beschrieben. Die Liste der so ermittelten Fälle ist eindrucksvoll: Alle Firmen waren in beträchtliche Betrügereien verwickelt. Die von Gerichten verhängten Bussen beliefen sich auf Summen von 93 Millionen bis 3,2 Milliarden US-Dollar. Ein Fall, der die öffentliche Gesundheit betraf, verursachte allein Ausgaben von mehreren Milliarden Dollar. Er wurde von einer parlamentarischen Kommission des Europarates aufgedeckt [2]. Goetsche schliesst daraus, dass Pharmafirmen in erheblichem Ausmass und wiederholt betrügerisch handelten und man darin eine Geschäftsstrategie sehen müsse. Das Vorgehen der Firmen zielte typischerweise auf erhöhten Gewinn, indem ihre Medikamente auch Patienten verschrieben werden sollten, für die sie 
behördlich nicht zugelassen waren. Die verschreibenden Ärzte wurden dabei zu wesentlichen, wenn auch oft unwissenden Komplizen des Marketings. Denn ohne ärztliche Verschreibung kein Verkauf. Eine Instrumentalisierung der Ärztschaft ist möglich, wenn die Opinion leaders der ärztlichen Spezialisten das Umfeld vorbereiten. Weltweit sind es akademische Mediziner, die in Fachzeitschriften und Fortbildungsveranstaltungen über einschlägige Studien berichten und daraus Therapieempfehlungen ableiten. Das ärztliche und das allgemeine Publikum vertraut ihnen, weil man, in "Treu und Glauben», davon ausgeht, dass sie unabhängige und kritische Experten des Themas sind. Als akademische Lehrer und Ärzte stehen sie im Ruf, im Interesse der Patienten zu handeln, ebenso wie die von ihnen angesprochenen Ärzte. Einerseits müssen Therapieempfehlungen auf Studien beruhen, wofür klinische Forscher, wie Dr. Matsubara, verantwortlich zeichnen. Andererseits bedürfen die Empfehlungen einer Ärztlichen und akademischen Autorität, die als (von Pharmafirmen) unabhängig erscheint.

Der Betrug wird also nur möglich durch Ärzte, die entweder unsorgfältig ausgeführte Studien publizieren, und/oder unkritisch solche Studien für verschreibende Ärzte kommentieren. Eine solch mehr oder weniger zustimmende, teils auch unbewusste Instrumentalisierung der Ärzteschaft geht weit über eine einfache Betrügerei hinaus, sie hat Korruptionscharakter [3-6]. Denn die Hauptbeteiligten, die Pharmaindustrie und unehrliche Wissenschaftler, können die Konsequenzen ihres Tuns nicht ignorieren: Einerseits bereichern sie sich auf Kosten der Allgemeinheit; ihr Betrug wird nur deshalb nicht bemerkt, weil ihnen das von Treu und Glauben geprägte Verhalten ihrer Umgebung und ihre soziale Stellung als Ärzte Schutz gewähren. Da aber andererseits auch sonstige Beteiligte und Mitwissende eher an Verheimlichung interessiert sein dürften, entwickelt sich um das fehlerhafte Verhalten eine «Mauer des Schweigens». Daher stellen die bekanntgewordenen Fälle wohl nur einen Teil der sicherlich viel vielschichtigeren Problematik dar.

\section{Public Health in der Schweiz}

Die Verzögerung, mit der in der Schweiz strukturelle Präventions-Massnahmen eingeführt werden, wird oft mit dem fragmentierten kantonalen Gesundheitssystem erklärt. Diese Erklärung ist nicht plausibel, wenn man die Tabakprävention mit einer anderen Herausforderung der Prävention vergleicht. Die AIDSEpidemie präsentierte sich je nach Kanton unterschiedlich komplex, dennoch war die Schweiz eines der ersten Länder, das durch pragmatische Zusammenarbeit die Ausbreitung des Virus unter Kontrolle brachte. Ein Beweis für die Behauptung: «Public health works, if there is political will», oder konkret: Die Experten überzeugten die Entscheidungsträger. Dagegen stellt man in der Tabakprävention fest, dass seit mehreren Jahren der Prozentsatz der Raucher nicht mehr abnimmt und dass der Anteil der ganz jungen Raucher und Raucherinnen sogar zunimmt. Das hat mit einer für die Tabakindustrie wenig einschränkenden Gesetzgebung zu tun $[7,8]$ : das Werbe- und Sponsoringverbot ist lückenhaft oder inexistent. Der parlamentarische Prozess für eine nationale Passivrauchgesetzgebung führte im Jahr 2008 schliesslich zu einem Alibi-Bundesgesetz [9], und die Volksinitiative für einen verbesserten Passivrauchschutz wurde mit Hilfe der Regierung, des Parlamentes, der Medien und der Marketingmaschinerie der Wirtschaft abgelehnt [10]. Die Schweiz gehört zu den wenigen Ländern der Erde, welche die WHO-Rahmenkonvention zwar unterschrieben, aber nicht ratifiziert haben [11]. Diese Konvention würde unser Land verpflichten, mit den andern Ländern zusammenzuarbeiten, um der weltweiten von multinationalen Firmen gesteuerten Tabakepidemie Einhalt zu gebieten.

Angesichts der enormen finanziellen Verluste, die durch wirksame strukturelle Präventionsmassnahmen für die in der Schweiz ansässige internationale Tabakindustrie entstehen würden, und der weltweiten Möglichkeiten ihrer Einflussnahme muss man annehmen, dass analog zu den Betrügereien der Pharmaindustrie, die Meinung von Experten auch hier eine Rolle spielt. Deren Einfluss widerspiegelt sich direkt in den Voten der Parlamentarier, von denen die Bevölkerung in "Treu und Glauben" annimmt, dass sie für das Gemeinwohl, in diesem Fall, für die öffentliche Gesundheit, einstehen.

\section{Der Fall Rylander}

Am 26. August 2003 fand die letzte einer Serie von Gerichtsverhandlungen statt, die mit der Verleumdungsklage des Wissenschaftlers und Umweltmediziners Ragnar Rylander zwei Jahre zuvor begonnen hatte. Die Präventionsaktivisten Diethelm und Rielle hatten den angesehenen Professor der Universität Genf in einer Pressekonferenz des Wissenschaftsbetrugs bezichtigt: Er hatte Forschungsresultate im Sinne seines Geldgebers zurechtgebogen und sich als unabhängig ausgegeben, obwohl er heimlich mehr als dreissig Jahre vertraglich für Philip Morris gearbeitet hatte [12]. Das Gericht sprach die Angeklagten vom Vorwurf der Verleumdung frei und hielt fest, dass Rylander «... nicht gezögert hatte, die Wissenschaft in den Dienst des Geldes zu stellen, obwohl die 
Aufgabe der Universität als öffentliche Einrichtung darin besteht, Wissen zu erlangen und zu verbreiten und die Lehrbeauftragten gegenüber der Gesellschaft in der Verantwortung stehen ...» [13].

\section{Lehren aus dem Fall Rylander}

Wie im Fall Matsubaras hatte Rylanders Verhalten Folgen für das Ansehen der universitären Institution. Aber auch für die Gesellschaft allgemein: Solange wissenschaftliche Zweifel an der Toxizität des Tabakrauchs bestanden, konnten weltweit keine staatlichen Passivrauchregelungen eingeführt werden.

\section{«Rylander war und ist kein Einzelfall.»}

Wie im Fall Matsubaras, haben die Medien Zusammenhänge vernachlässigt, die der Prozess ans Licht gebracht hatte: Rylander war und ist kein Einzelfall. Die während des Prozesses von Diethelm und Rielle vorgelegten öffentlich zugänglichen Dokumente der Tabakindustrie beweisen, dass sie ihren Einfluss auf den Wissenschaftsbetrieb auch in der Schweiz mit Methode organisierte [14]. Ärzte wurden in einem «white coat program" genannten Projekt in die systematische Desinformation einbezogen [15]. Th. Grüning deckte durch Analyse von Industrie-Dokumenten [16] mehr als ein halbes Dutzend Fälle heimlicher Zusammenarbeit zwischen hochgestellten medizinischen Autoritäten und der Tabakindustrie Deutschlands auf, die auch in der Presse kommentiert wurden [17].

Kürzlich folgerte eine wissenschaftliche Arbeit der Universität Zürich, dass die Einführung der neutralen Zigarettenpackung keinen Einfluss auf das Rauchverhalten der Jungen hätte [18]. Die angewendete statistische Methode wurde als inadäquat kritisiert $[19,20]$ und man entdeckte, dass Philip Morris nicht nur die Datenbasis zur Analyse geliefert hatte, sondern auch durch den Finanzierungsvertrag der Studie sich das Recht ausbedungen hatte, bei der Redaktion der wissenschaftlichen Arbeit mitzureden [21].

Obwohl die Anschuldigungen gegen Rylander auf öffentlich zugänglichen Dokumenten der Tabakindustrie beruhten, hatten Justiz und Universität Mühe, den Tatsachen ins Auge zu sehen [22]. Es fällt auf, dass die Kenntnis des Falls Rylander und seiner politischen Zusammenhänge für die Schweiz auch bei Ärzten und in Gesundheitskreisen gering sind. Hier muss man die Abhängigkeit der Medien, auch der Medizinischen Fachmedien, von Wirtschaftskreisen als Grund für die fehlende Neugier der Informationsfachleute vermuten. Die Finanzierung der Universitäten $[23,24]$ wird zwar erneut thematisiert, aber die Forde- rung nach Transparenz in dieser fundamentalen Frage wird auch von den Universitäten nur zögernd behandelt [25-27]. Der grosse Druck auf Prävention und öffentliches Gesundheitswesen, den man seitens der Tabakindustrie logischerweise vermuten muss, wird in dieser Debatte kaum beleuchtet. Dies, obwohl die Argumente der Exponenten der öffentlichen Gesundheit im Zusammenhang mit der Passivrauchinitiative, der Abstimmung über das Präventionsgesetz und der Vernehmlassung zum Entwurf des Tabakproduktegesetzes in der öffentlichen Diskussion als Experten zu vernehmen sein sollten. Um die Tabakprävention und ihre Interessenkonflikte hat sich, wie im Medikamentenmarkt, eine Mauer des Schweigens aufgebaut.

\section{Die Rolle der Experten und die Passivrauchschutzinitiative}

Das Bundesgesetz zum Passivrauchschutz entstand, nachdem der Parlamentarier Prof. Felix Gutzwiller, damals Vorsteher des Institutes für Sozial- und Präventivmedizin der Universität Zürich, 2004 eine parlamentarische Initiative lanciert hatte, die rauchfreie Arbeitsplätze für alle vorsah. Der parlamentarische Prozess demontierte danach etappenweise den Vorschlag der Gesundheitskommission, so dass der prinzipielle Schutz vor Passivrauch im Bundesgesetz von 2008 durch gravierende Ausnahmen eingeschränkt wurde: Falls der Gastraum kleiner als $80 \mathrm{~m}^{2}$ ist, darf der Wirt entscheiden, ob sein Lokal rauchfrei sein soll oder nicht. Raucherräume sind grundsätzlich nicht bedient, ausser die Angestellten stimmen schriftlich im Arbeitsvertrag ihrer Verräucherung zu. Das Bundesgesetz gilt in der ganzen Schweiz ausser in Kantonen mit strengeren Regeln. Eine Allianz von Gesundheits- und Ärzteorganisationen lancierte darauf, der Lungenliga folgend, die Volksinitiative "Schutz vor Passivrauch» für eine wirksamere nationale Gesetzgebung.

Dass das Alibi-Bundesgesetz von 2008 unter dem Druck der Wirtschaftslobby zustande gekommen war, ist offensichtlich: Es wurde mit einer Stimme im Nationalrat und nur durch Stichentscheid des Ständerat-Präsidenten Christoffel Brändli (damals santésuisse-Präsident) angenommen. Anders als im Kampf gegen die AIDSEpidemie, kommunizierten die Vertreter der öffentlichen Gesundheit nicht wirksam mit den Entscheidungsträgern. Die Bevölkerung hätte in «Treu und Glauben» von ärztlichen Experten erwarten dürfen, dass sie im Vorfeld der Volksabstimmung zur Passivrauchinitiative laut und vernehmlich für eine wirksame Gesetzgebung eintreten. Ihr Schweigen wird in der nächsten Ausgabe der SÄZ näher beleuchtet. 